\title{
Gioielli, acconciature ed abiti nella Mantova romana
}

\author{
Giovanna Ziliani
}

I ritratti maschili e femminili di età romana rinvenuti a Mantova ci forniscono un quadro abbastanza completo circa i costumi, le acconciature e i gioielli dell'epoca, sia maschili che femminili, dato che questi vengono raffigurati su quasi tutte le sculture. Si tratta in particolare di immagini funerarie, nate come ornamento individualizzante di segnacoli grandi e piccoli, realizzate tutte tra la fine del I secolo a.C. e la prima metà del I secolo d.C.

Sfortunatamente si è conservato un solo ritratto a figura completa, quello di Acutia Mater ${ }^{1}$, mentre per quanto rigurda le altre immagini abbiamo tre busti di togati acefali, teste singole, maschili e femminili, e figure a mezzo busto o rappresentate solo fino alla base del collo, poste all'interno di nicchie su stele. Il ritratto di Acutia si presenta particolarmente interessante non solo perché quasi completamente intatto, ma anche perché presenta caratteristiche uniche e particolari. L'immagine nel complesso mostra una figura di donna, tendenzialmente idealizzata nei tratti del volto ma connotata da dettagli ornamentali che dovevano essere propri del personaggio raffigurato; al collo infatti notiamo un doppio giro di collana a maglie avvitate, decorata da un grosso pendente a foglia o a conchiglia che trova riscontro nella moda di fine I secolo a.C.-inizi I secolo d.C. ${ }^{2}$; ai lobi si notano orecchini a goccia. Le braccia sono ornate da armille lisce e all'anulare destro si vede un anello a fascia scarsamente conservato.

Significativa è la raffigurazione delle vesti, che prevedono una tunica con scollo rotondo, utilizzata come indumento intimo, al di sopra della quale è posta una seconda tunica, a manica lunga, con scollo a "V". Sopra le vesti vengono indossati contemporaneamente la palla, che avvolge le spalle e la vita, scendendo lungo un fianco, e un corto velo posto a coprire il capo. La consueta immagine capite velato prevede canonicamente la copertura della testa con un lembo della palla, unico si presenta quindi il caso mantovano, che vede due elementi separati, la palla e il velo, per rappresentare un'immagine comune nella ritrattistica ufficiale. È da ravvisare in questo la presenza di una moda locale di tipo provinciale, che denuncia un' accettazione libera e non pedissequa della moda e dei costumi della capiatale.

Caratteristica è infine la presenza, ad un angolo del velo, di un gioiello a goccia, che si ritrova identico nella terminazione inferiore della toga di un ritratto maschile ${ }^{3}$, sicuramente appartenente ad un personaggio della medesima famiglia di Acutia. L'acconciatura della matrona presenta i caratteri tipici della primissima età imperiale, che prevede capelli spartiti al centro da una scriminatura centrale e nodus frontale.

Ispirate allo stile tardo repubblicano sono le toghe maschili indossate da tre personaggi, i cui ritratti sono forse databili alla primissima età imperiale; i togati si

\footnotetext{
${ }^{1}$ St. 11102 per molti aspetti ricorda la ben più tarda "matrona ammantata" di Pergola interpretata come Livia (Stucchi 1988), la quale presenta ugualmente il capo velato e l'acconciatura con sciminatura centrale terminante al di sotto della stola. Ancora più stringente risulta il confronto con un ritratto femminile di Padova, datato al primo decennio del I sec. d.C. (Ghedini 1975, pp. 361-372) anch'esso certamente ispirato ai ritratti ufficiali di Livia.

${ }^{2}$ Per quanto riguarda la moda di I secolo d.C. si vedanogli esemplari di collana rinvenuti a Pompei (Carducci 1962, pp. 160, 162, 164, tavole LXIII e LXIV; Pirzio Biroli 1992, pp. 106, 107, 111).

${ }^{3}$ St. 11110 .
} 
presentano due stanti, uno seduto ${ }^{4}$. Le toghe dei personaggi stanti presentano un ampio sinus che termina fino sotto l'ombelico e non consente di sorreggere il braccio, che passa all'interno del lembo di stoffa, mentre per quanto riguarda il togato seduto, la toga presenta un sinus più corto e aderente al corpo, che si attorciglia al braccio quasi sospendendolo al collo. Le toghe dei primi due personaggi sono lunghe sino sotto il ginocchio ma lasciano scoperte le caviglie, e in una la lacinia lambisce la caviglia sinistra ${ }^{5}$ presentando una terminazione con pendente a goccia. Pessimamente conservata è la parte inferiore del togato seduto, che risulta praticamente privo delle gambe, mentre interessante per quanto riguarda i due togati stanti è la presenza dei piedi, presso i quali sono sommariamente tracciati dei calzari alti. Sfortunatamente tutte e tre le statue si presentano acefale, non è quindi possibile associare ad esse volti ed acconciature.

Per quanto riguarda ancora la moda maschile troviamo a Mantova anche un ritratto a mezzo busto posto all'interno di una stele ad archetto di tipo opitergino, questa, essendo appunto un prodotto di importazione, non si ritiene particolarmente significativa per quanto riguarda strettamente la moda mantovana, anche se fornisce comunque importanti informazioni circa i capi di abbigliamento, le acconciature e i gioielli presenti in aree limitrofe al mantovano come appunto il Veneto, che vedeva in Mantova uno tra i principali acquirenti di segnacoli funerari e materiale lapideo. Il ritratto maschile della stele opitergina presenta una toga con sinus ampio, paragonabile a quello visto per i due togati stanti; in questo caso il corpo è associato al ritratto, che raffigura un uomo anziano dalla folta capigliatura leggermente ondulata, con ciocche pettinate in avanti a formare una spessa frangia. Alla mano sinistra, che trattiene un lembo della toga, si nota un anello con sigillo, indossato al dito mignolo; questo è probabilmente caratteristico del personaggio e simbolo dell'ottenimento di una qualche carica pubblica. La figura maschile è accompagnata da due figure femminili, certamente moglie e figlia del personaggio rappresentanto. Interessante è notare come tra la madre e la figlia vi sia una differenza sia nell'abbigliamento che nell'acconciatura, cosa che denota il mantenimento da parte delle persone più anziane di costumi in voga nei decenni precedenti. L'abito e i capelli della madre si ispirano, come già quelli del padre, ad una moda tardo repubblicana-primo augustea, la donna presenta infatti, come Acutia, un'acconciaura con sciminatura centrale e capo coperto dalla palla; la figlia mostra invece una più moderna acconciatura ad onde e boccoli che permette di datare la stele ai primi due decenni del I secolo d.C.

Una simile acconciatura ad onde e boccoli si trova anche in altri ritratti femminili del mantovano, in particolare nell'elaborata testa di Pietole ${ }^{6}$, ma anche nelle due stele di Cassia e di Sentia ${ }^{7}$, queste ultime prodotte in serie da un unico modello. La testa femminile di Pietole sfoggia un'accurata acconciatura in cui si riconosce il

\footnotetext{
${ }^{4}$ St. 11109 mostra affinità con il togato di Maccaretolo (Negretto 2005, pp. 161-198) datato fine I sec. a.C.-inizi I sec. d.C.; ugualmente St. 11110 può trovare confronto in questo ritratto e con altri datati alla fine del I sec. a.C., che mostrano lo stesso modello di toga. I.G. 6604 presenta somiglianze sia con una statua rinvenuta a Brescia, datata all'inizio del I sec. d.C. (Morandini-Stella 1998, p. 69) che con un togato seduto trovato a Vicenza (Zerbinati 1987, p. 251) e datato fine I sec. a.C.-inizio I sec. d.C.

${ }^{5}$ St. 11110 .

${ }^{6}$ I.G. 6926 presenta somiglianze con ritratti altinati (Scarpellini 1987, nn ${ }^{\circ} 11-12$, p. 129, figg. 14-15, tav. 10), datati ad età augusteo-tiberiana. I volti femminili hanno labbra carnose e sguardi dolenti; un incarnato morbido che contrasta con i panneggi delle vesti e con la lavorazione delle ciocche sottili ed ondulate dei capelli.

${ }^{7}$ I.G. 12160 e I.G. 12154 sono chiaramente prodotte dalla medesima officina, come si deduce osservandone le caratteristiche e le dimensioni; il ritratto utilizzato come modello presenta un'acconciatura diffusa durante il I sec. d.C., mentre il taglio del busto, secondo Mansuelli (Mansuelli 1966, p. 53), conferma la medesima datazione.
} 
lavoro del trapano, che consente una maggior cura dei dettagli. Quattro grossi boccoli avvitati scendono ai lati del collo e si posano sulle spalle, mentre la scriminatura sopra la nuca spartisce l'intera capigliatura in due masse rigofie che formano quattro morbide onde su ciascun lato. Dell'abito si riconoscono la tunica, dallo scollo rotondo, e la palla, le cui pieghe pendono dalla spalla sinistra. Alle orecchie sembra di poter riconoscere piccoli orecchini globulari. Simile, anche se pessimamente conservata, doveva essere l'acconciatura di Sentia Maxuma e Cassia Tertia, nei cui ritratti è riscontrabile una traccia di lunghi boccoli che scendevano sino allo sterno. Erano presenti anche orecchini globulari, ampiamente in voga durante tutta l'età imperiale, mentre i busti, realizzati sino all'altezza dello sterno, sembrano essere privi di indumenti. Identica acconciatura ad onde e boccoli poteva forse essere presente anche nel ritratto proveniente da Acquanegra sul Chiese, che però si presenta molto lacunoso. Per quanto riguarda quest'ultimo, al lobo dell'unico orecchio ancora visibile non si nota la presenza di orecchini.

Gli ultimi ritratti considerati mostrano caratteri stilizzati, derivanti da una produzione in serie, che talvota ci fornisce solo indicazioni generali circa la moda dell'epoca in cui sono stati prodotti; le ultime due immagini di cui si vuole trattare denunciano in modo ancora più evidente la presenza di una produzione a basso costo, che si avvale di modelli semplici e universali, validi per qualsiasi committente. È questo il caso della stele di Lucius Sentius ${ }^{8}$, che ci presenta un generico volto maschile, realizzato in maniera rigida e sommaria. L'acconciatura a calotta si presenta appiattita sul capo e mostra un'attaccatura cuoriforme che termina con una profonda punta al centro della fronte. Come nel caso di Sentia e Cassia, il busto è rappresentato nudo e terminante appena al di sotto dello sterno.

Un'ultima immagine tipizzata è quella che si ritrova nella stele di bambina ${ }^{9}$, che rappresenta un generico volto infantile con capello corto a frangia. Solo l'iscrizione ci permette di identificare il sesso della defunta, i cui capelli a calotta sono solcati da piccole incisioni atte a rendere le strette ciocche parallele tra loro. I capi di abbigliamento sono resi mediante sottili linee incise che non ne permettono una puntuale identificazione. Anche se il ritratto di bambina si mostra generico sia nell'acconciatura che nella rappresentazione dei capi di vestiario, la stele di Septumia è comunque per noi una preziosissima fonte iconografica per quanto riguarda la moda in generale, dato che sui due lati del cippo si trovano rappresentati due giocolieri. Si tratta verosimilmente di due schiavi, l'uno dei quali indossa solamente un subligar legato strettamente in vita, mentre l'altro, raffigurato sul lato opposto, sfoggia una corta tunica senza maniche, legata in vita da una fascia. Probabilmente in ragione della condizione servile, entrambi i personaggi si presentano scalzi. La raffigurazione dei due schiavi in relazione ai personaggi di rango visti precedentemente risulta particolarmente significativa inquanto ci dimostra come la raffigurazione di ornamenti e capi d'abbigliamento servisse, nel mondo romano, come elemento di immediata distinzione e riconoscimento del rango di appartenenza, risultando sostitutiva o eventualmente accessoria alla presenza del testo epigrafico.

\footnotetext{
${ }^{8}$ Il ritratto presente su I.G. 12151 presenza una notevole somiglianza con un ritratto maschile rinvenuto a Brescia (Morandini-Stella 1998, p. 74), datato al I sec. d.C.; simili sono la forma del viso, il collo e le orecchie appiattite ai lati del capo; simile è anche l'acconciatura con ampia stempiatura cuoriforme. Il taglio del busto e la rigidità dei lineamenti confermano la datazione di inizio I. sec. d.C. (Mansuelli 1966, p. 53).

${ }^{9}$ Un chiaro confronto con la stele di Septumia si ha in una stele di bambino rinvenuta a Ferrara (Mansuelli 1966, $\mathrm{n}^{\circ} 15$, fig. 24, p. 128) sulla quale si trova ugualmente raffigurato un coniglietto.
} 


\section{Catalogo:}

\section{Satua femminile}

\section{St. 11102}

Scavo del Seminario, via Cairoli, 1971, Mantova.

Pietra di Vicenza (Zezza 1982).

Altezza totale prima del restauro142 cm; dopo il restauro, h. $178 \mathrm{~cm}$; largh. $46 \mathrm{~cm}$; spess. $32 \mathrm{~cm}$

Ultimo decennio I sec. a.C.

La figura femminile, ricomposta da due pezzi, è priva dei piedi e della base che doveva sostenerla. Veste una tunica a maniche lunghe che scende fino alle caviglie, e la stola, trattenuta sul fianco sinistro dalla mano destra. Il capo è coperto da un corto velo che scende sulle spalle. Al collo porta una collana a maglie avvitate, che compie due giri attorno al collo e termina sul petto con un pendente a foglia o conchiglia; ai polsi vi sono tracce di armille; all'anulare destro porta un anello, alle orecchie orecchini globulari.

La capigliatura presenta scriminatura centrale e capelli raccolti dietro la nuca, al di sotto del velo. Il volto, fortemente lacunoso, è caratterizzato da occhi di forma ovulare e palpebre a cordone.

\section{Bibliografia}

TAMASSIA 1976, pp. 127-134; 1980, pp. 137-155; BOSI 1983, pp. 97-115; TAMASSIA 1984, pp. 89-94.

\section{Testa maschile}

\section{St. 11103}

Scavo del seminario, via Cairoli, 1971, Mantova.

Pietra di Vicenza (Zezza 1982).

h. $35 \mathrm{~cm}$, largh. max. $21 \mathrm{~cm}$, spess. max. $30 \mathrm{~cm}$ c.a.

Ultimo decennio I sec. a.C.

Ritratto maschile che si conserva fino all'altezza dello sterno e per un brevissimo tratto della spalla destra. Posteriormente la testa ha una sporgenza dello spessore di 7 $\mathrm{cm}$ c.a., per una larghezza di $10 \mathrm{~cm}$. La parte superiore del capo sembra essere stata appiattita. La capigliatura è resa mediante brevi solchi che rendono, grazie all'effetto chiaroscurale, le ondulazioni della pettinatura, con le ciocche pettinate in avanti a formare un arco sulla fronte. Il viso è largo e piatto, con arcate sopracciliari massicce e sporgenti; gli occhi, di forma ovulare, presentano palpebre cordonate; il naso è largo, la bocca ha labbra sottili con solchi ai due lati e con fessura infralabbiale profondamente incisa. Il mento è massiccio e squadrato, le guance lievemente incavate. Le orecchie sono grandi ed asimmetriche, diverse per forma e dimensione; l'orecchio destro ha attaccatura più alta rispetto al sinistro. 
TAMASSIA 1974, pp. 127-134; 1980, pp. 137-155; BOSI 1983, pp. 97-115.

\section{Testa maschile}

\section{St. 11104}

Scavo del seminario, via Cairoli, 1971, Mantova.

Pietra di Vicenza (Zezza 1982).

h. $29 \mathrm{~cm}$ c.a.

Ultimo decennio del I sec. a.C.

Ritratto maschile lacunoso nella parte inferiore del viso; si conserva fino alla base del collo ed è completamente privo di naso, mento, bocca. I capelli, resi mediante una calotta solcata da incisioni parallele, sono pettinati con la frangia girata verso destra; ai lati i capelli scendono sulle tempie a formare due corte basette. Le orecchie sono asimmetriche, la destra è infatti posizionata più in alto rispetto alla sinistra; sono inoltre diverse per forma e dimensioni. Le arcfate sopracciliari sono taglienti ed arcuate, gli occhi allungati ed ovulari, con palpebre a cordone. Il naso doveva essere abbastanza largo. La bocca doveva avere probabilmente due solcature ai lati. Delle labbra non si conserva nulla, resta solo un profondo solco che andava a costituire la fessura intralabbiale. Il viso, in generale, ha forma allungata, con guance piatte e lineamenti marcati, resi in modo molto grossolano. Il collo è massiccio. Presenta tutti i caratteri propri del ritratto provinciale.

Bibliografia

TAMASSIA 1976, pp. 127-134; 1980, pp. 137-155; BOSI 1983, pp. 97-115.

\section{Statua togato}

\section{St. 11109}

Scavo del seminario, via Cairoli, 1971, Mantova.

Pietra di Vicenza (Zezza 1982).

Prima del restauro h. max. cons. $130 \mathrm{~cm}$; dopo il restauro h. cm 176 c.a., largh. $53 \mathrm{~cm}$ c.a., spess. $38 \mathrm{~cm}$ c.a.

Ultimo decennio del I sec. a.C.

Statua frammentaria e acefala di togato, ricomposta da due pezzi combacianti; la mano destra esce dalla toga per trattenere il sinus che ricade sulla spalla sinistra; il braccio sinistro è lievemente piegato e con la mano socchiusa per trattenere il rotulo. La statua si conserva fino all'altezza delle ginocchia. Il lavoro è molto grezzo; le piegature della veste sono date da una serie di linee incise con andamento circolare. Le mani sono squadrate, con dita rettangolari. L'andamento del corpo lascia presumere che la gamba destra scartasse di lato, mentre la sinistra doveva costituire la base d'appoggio per il peso. La statua doveva essere fatta per essere vista esclusivamente dal davanti, dato che il retro è sommariamente abbozzato e piatto. 
TAMASSIA 1976, pp. 127-134; 1980, pp. 137-155; BOSI 1983, pp. 97-115.

\section{Statua togato}

\section{St. 11110}

Scavo del seminario, via Cairoli, 1971, Mantova.

Pietra di Vicenza (Zezza 1982).

Due frammenti: h. max. cons. rispettivamente $61 \mathrm{~cm} \mathrm{e} 73 \mathrm{~cm}$; h. tot dopo il restauro $183 \mathrm{~cm}$, largh. $48 \mathrm{~cm}$ c.a., spess. $33 \mathrm{~cm}$ c.a.

Ultimo decennio del I sec. a.C.

Due frammenti non ricomponibili forse di una stessa statua di togato. La parte superiore, acefala, presenta il braccio destro che afferra il sinus sulla sinistra; del braccio sinistro si conserva solamente l'attaccatura. Il frammento inferiore comprende i piedi, con la gamba destra leggermente flessa verso l'esterno e la lacinia che scende a lambire la caviglia sinistra. In fianco al piede sinistro si trova una piccola capsa cilindrica decorata. Le pieghe della toga sono incise con profonde solcature e scendono rigide lungo il corpo; la veste sembra aderire al ginocchio destro, ma si è ben lontani dall'ottenere l'effetto di plasticità. Il lavoro è molto grezzo ed il retro della statua si presenta come un blocco sommariamente sbozzato ed appiattito.

Bibliografia

TAMASSIA 1976, pp. 127-134; 1980, pp. 137-155; BOSI 1983, pp. 97-115.

\section{Togato seduto}

\section{I.G. 6604}

Ignota. Forse proviene da Mantova città.

Tufo vicentino (ATSAL).

h. max. cons. $140 \mathrm{~cm}$, largh. $63 \mathrm{~cm}$, spess. $37 \mathrm{~cm}$.

Ultimo decennio del I sec. a.C.

Statua di togato seduto, acefala, priva delle gambe e della mano sinistra; si riconosce bene il tipo di toga tardo repubblicana-primo imperiale, dalla quale esce la mano destra che trattiene il sinus. Le pieghe sono incise con solchi profondi che danno un grossolano effetto chiaroscurale; anche la piega del sinus al di sotto del polso destro si svolge rigidamente, con solcature " a gomito" che tentano di imitare la flessuosità del panneggio. la mano conservata si presenta squadrata, con dita rettangolari e rigide. Sul lato destro della statua si riconosce lo sgabello, simbolo della carica pubblica ricoperta in vita dal personaggio.

Bibliografia

TAMASSIA 1995, pp. 281-283. 


\section{Ritratto Femminile}

\section{St. 16325}

Ignoto. Acquanegra sul Chiese, 1963. Murata nella soffitta di un privato, in vicolo Arfini. Racuperata dal sig. Gianluigi Arcari il 28 febbraio 1967 e consegnata alla Soprintendenza della Lombardia. Attualmente conservata a Mantova.

Marmo.

h. max. cons. $27 \mathrm{~cm}$, largh. $24,5 \mathrm{~cm}$, spess. $10,5 \mathrm{~cm}$.

I sec. d.C.

Scalpellata nella parte posteriore, forse lavorata a bassorilievo; non sembrano conservarsi tracce della scriminatura centrale. Sembra che il viso femminile fosse incorniciato da una pettinatura rigonfia ai lati del capo a formare tre onde; dei due orecchi si conserva solo il sinistro, al cui lobo non si riscontra la presenza di orecchini. Con il restauro è stato asportato uno strato di colore nero che ricopriva gran parte del volto. L'acconciatura trova confronto con le acconciature di inizi I sec. d.C. visibili sul ritratto di donna giovane della stele opitergina (senza numero) e sulla testa femminile da Pietole (I.G. 6926), così come nei ritratti di Cassia (I.G. 12160) e Sentia (I.G. 12154).

Bibliografia

DALL'ACQUA 1969, p. 329-354.

\section{Ritratto Femminile}

\section{I.G. 6926}

Proveniente da Virgilio, frazione di pietole, zona del forte. Venne donata al Museo Civico dal dott. Cesare Bonoris nel 1866-67, poi passata a Palazzo Ducale nel 1915.

Marmo bianco a grana fine (ATSAL).

h. max. cons. $35,5 \mathrm{~cm}$, altezza volto $24 \mathrm{~cm}$.

Prima metà del I sec. d.C.

Il retro della testa presenta scalpellature grossolane, forse causate da un successivo adattamento ad una nicchia in un monumento funerario. La capigliatura è scriminata la centro con ampie ondulazioni; le ciocche sono rese mediante solcatura, assumendo però, in generale, una certa plasticità. I capelli coprono le orecchie e sono raccolti dietro la nuca; da qui scendono sulle spalle in quattro grossi boccoli a vite, resi mediante profonde incisioni che offrono un marcato effetto chiaroscurale; i particolari sono lavorati a trapano. Il volto è caratterizzato da lineamenti marcati e a tratti taglienti, mentre un leggero effetto plastico si può notare nelle guance. L'intento è forse quello di una resa naturalistica, anche se la realizzazione è ancora abbastanza grossolana e di stampo chiaramente provinciale. Gli occhi sono di forma ovulare, compresi fra palpebre a cordone; il mento è ampio e prominente, le guance appiattite. Il collo è ben tornito e su di una spalla si conserva quello che sembra essere il lembo di una palla dalle pieghe circolari, discendente sullo scollo della tunica.

Bibliografia 


\section{Stele con ritratto femminile}

\section{I.G. 12160}

Nel XV secolo si trovava murata presso l'antica porta della città, nei pressi delle carceri, forse nella zona poi denominata "stabbio", infine passata a Palazzo Ducale dove si trova attualmente.

Bronzetto di Verona (Zezza 1982).

h. $\max$. cons. $91,5 \mathrm{~cm}$, largh. $57 \mathrm{~cm}$; spess. $11,5 \mathrm{~cm}$.

(C.I.L. V, 4072) Cassiae L(ucii) f(iliae)/Tertiai matri. Capitale quadrata, solco a sezione angolare, interpunzione triangolare. Linea I $8,5 \mathrm{~cm}$, linea II $6,2 \mathrm{~cm}$.

Fine I sec. a.C.

Realizzata dalla stessa maestranza che ha prodotto la stele di Sentia Maxuma. La stele centinata presenta una nicchia con doppia cornice a gola al cui interno si trova un busto femminile rappresentato sino all'altezza delle clavicole. La figura presenta un collo tornito "a scafo di nave" a si impianta sul busto mediante una linea netta; lo sterno si flette verso il basso ed ha un taglio tondeggiante. La capigliatura ad onde e boccoli è quella tipica del periodo a cavallo tra l'ultimo decennio del i sec. a.C. e il primo decennio del I secolo d.C. L'iscrizione è breve; le superfici sono spianate a martellina. Nella parte alta le superfici sono arrotondate e smussate a circa tre quarti del segnacolo, mentre a partire dal basso troviamo un piccolo gradino che divide la parte superiore della stele da quella inferiore, che presenta superfici più squadrate. Ampiamente restaurata, presenta molte integrazioni fatte con gesso.

Bibliografia

TAMASSIA 1965, p. 84.

\section{Stele con ritratto femminile}

\section{I.G. 12154}

Ignoto. Conservata all'interno della Cattedrale di S. Andrea a Mantova, presso la cappella dell'incoronata, poi passata al Museo dell'Accademia e quindi a Palazzo Ducale nel 1915.

Bronzetto di Verona (Zezza 1982).

h. max. cons. $89 \mathrm{~cm}$, largh. $57,5 \mathrm{~cm}$, spess. $29,5 \mathrm{~cm}$.

(CIL V, 4080) Sentiai C(ai) f(iliai)/Maxumai/uxori. Capitale quadrata con sezione angolare, linea I $8,5 \mathrm{~cm}$, linea II $6,2 \mathrm{~cm}$, linea III $6 \mathrm{~cm}$.

Fine I sec. a.C.

Blocco rettangolare, probabilmente parte di un segnacolo di dimensioni maggiori, squadrato. Presenta nella parte superiore una nicchia centinata con cornice a gola. Le superfici sono spianate a martellina. Del ritratto si conserva un busto, riconoscibile come femminile dalla massa della capigliatura, gonfia ai lati del capo, e dalla presenza 
dei lunghi boccoli, che scendono davanti alle spalle. Si nota la presenza di orecchini globulari. Il mento è fine ed allungato, mentre illeggibili risultano i lienamenti del volto. Si intuisce una discreta resa plastica, denunciata da un collo ben tornito e da spalle che seguono linee sinuose. La superfice su cui si svolge lo specchio epigrafico è liscia e posta al di sotto della nicchia. Nella parte superiore del segnacolo si nota un foro per grappa, probabilmente per l'impianto di ante in legno.

Bibliografia

TAMASSIA 1965, p. 80.

\section{Cippo a nicchia quadrata}

\section{CIL V 2688}

Ignoto.

Calcare grigio.

h. max. cons. $72 \mathrm{~cm}$, largh. $41 \mathrm{~cm}$, spess. $37 \mathrm{~cm}$.

(CIL V, 2688) Septumia o.../spica anno/ et mense tertio. Lettere irregolari, linea I 4 $\mathrm{cm}$, linea II $4 \mathrm{~cm}$, linea III $3 \mathrm{~cm}$, linea IV $3 \mathrm{~cm}$.

Inizi I sec. d.C.

Cippo rettangolare coronato alla sommità dallo specchio epigrafico. Lo specchio epigrafico poggia su due pilastrini lisci con piccoli capitelli a listello. Al centro si trova la nicchia quadrata $(29,3 \times 29,3 \mathrm{~cm})$ contenente il ritratto della piccola defunta. Al di sotto si svolge lo specchio epigrafico, liscio e di forma rettangolare. Il volto infantile è rappresentato con capelli corti e lisci che scendono sulla fronte e sulle tempie, pettinati in avanti. La capigliatura a calotta è solcata da piccole e profonde incisioni. Le orecchie sono piccole e appiattite ai lati del capo; il volto presenta un'espressione dolente, con occhi piegati verso il basso e la bocca stretta, senza sorriso. Il viso tondo si innesta su un collo di forma cilindrica, allungato e ben tornito, a sua volta innestato su una geometrica base di appoggio data da spalle appiattite e spioventi. Il ritratto emerge di tre quarti dalla nicchia. Due sottili linee oblique potrebbero indicare lo scollo di un indumento. Al di sotto dello specchio epigrafico è rappresentato un leprotto in rilievo, in posizione accucciata e con le lunghe orecchie tirate all'indietro. Entrambi i lati del segnacolo sono decorati da figure di giocolieri; sul lato sinistro si vede un acrobata scalzo e cinto da un subligar, che si esibisce con sei palline; sul lato destro si trova invece un giocoliere vestito con una corta tunica senza maniche, allacciata in vita da una fascia, il quale si esibisce con sette palline. L'intero corpo del cippo poggia su uno zoccolo liscio. Sui due lati anteriori del segnacolo, nella parte inferiore, si notano due fori per grappa della larghezza di $2 \mathrm{~cm}$ c.a., perfettamente simmetrici, certamente connessi alla presenza di antine in legno.

Bibliografia

ZILIANI 2001, pp. 91-99. 


\section{Stele di tipo opitergino}

\section{Senza numero}

Ignoto. Conservata presso i depositi di Palazzo Ducale.

Calcare grigio.

h. max. cons. $67 \mathrm{~cm}$, largh. $64 \mathrm{~cm}$, spess. $19 \mathrm{~cm}$.

Inizi I sec. d.C.

Stele a nicchia incurvata, coronata da un frontone modanato. Gli acroteri, a forma di leoncini accovacciati con la bocca spalncata, sono privi della metà posteriore. Gli spioventi del frontone poggiano su due pilastrini lisci con capitellini a listello. La stele poggia su un basso podio, leggermente aggettante. All'interno della nicchia, partendo da sinistra verso destra rispetto all'osservatore, trovano posto tre ritratti, due femminili e uno maschile. L'uomo presenta una folta capigliatura, con ciocche mosse al di sopra del capo e tirate all'indietro sulle tempie. Le orrecchie sono piccole e sporgenti ai lati del capo; il volto presenta tratti spigolosi e profonde righe ai lati della bocca; il personaggio indossa una toga con sinus, trattenuta dalla mano sisnistra, presso la quale notiamo un anello al dito mignolo. Gli abiti sono resi mediante numerose solcature e pieghe. A sinistra dell'osservatore si trova il busto di una donna giovane con capelli gonfi ai lati del capo, legati dietro la nuca e ricadenti in due lunghi boccoli ai lati del collo. Il viso tondo presenta una bocca stretta e carnosa, gli occhi sono ovulari e contornati da palpebre a cordone. Al centro troviamo il ritratto di una donna anziana dal capo velato; la stola che scende sulle spalle è trattenuta dalla mano destra. Il volto, magro e allungato, mostra due evidenti rughe ai lati della bocca, stretta e allungata.

Bibliografia

Inedito.

\section{Stele a nicchia centinata}

\section{I.G. 12151}

Ignoto. Forse rinvenuta nel 1753 nell'ala del casino degli Angeli, fuori Porta Pradella, secondo altri riutilizzata nella porta rustica del palazzo del conte Marcello Donati.

Bronzetto di Verona (ATSAL).

h. max. cons. $125 \mathrm{~cm}$, largh. $46,5 \mathrm{~cm}$, spess. $18,5 \mathrm{~cm}$.

(CIL V, 4079) L(ucius) Sentius/C(ai) f(ilius) Ani(ensi tribu). Caratteri regolari, linea I $6 \mathrm{~cm}$, linea II $6 \mathrm{~cm}$ con $i$ longa che misura $8 \mathrm{~cm}$. Interpunzioni triangolari.

Inizi I sec. d.C.

Lavorata a martellina, la stele si presenta centinata, con nicchia ad arco all'interno della quale è ricavato il ritratto, che sporge dal fondo per un terzo. Al di sotto della nicchia si trova l'epigrafe che si svolge su due linee. Il personaggio maschile è rappresentato fino alle spalle, rappresentate in modo sommario e geometrico. Il volto è mal conservato e i lineamenti risultano illeggibili anche se risulta evidente che la lavorazione doveva essere grossolana. Collo, spalle e busto sono rigidamente quadrati; il viso ha una forma rettangolare; i capelli sono una calotta compatta con attaccatura cuoriforme che lascia ampiamente scoperte le tempi. Le orecchie sono 
aderenti al capo e attaccate al di sopra della linea degli occhi. La superficie al di sotto della nicchia è levigata.

Bibliografia

TAMASSIA 1965, p. 80.

Abbreviazioni bibliografiche:

ATSAL

BOSI 1983

CARDUCCI 1962

DALL'ACQUA 1969

GHEDINI 1975

MANSUELLI 1966

MORANDINI-STELLA 1998

NEGRETTO 2005

PIRZIO BIROLI 1992

SCARPELLINI 1987

STUCCHI 1988

TAMASSIA 1965

TAMASSIA 1968

TAMASSIA 1976

TAMASSIA 1980

TAMASSIA 1984
Archivio topografico Soprintendenza Archeologica della Lombardia.

N. Bosi, Considerazioni storiche su alcune stele ed iscrizioni d'età romana rivenute a Mantova in "Atti e memorie dell'Accademia Virgiliana di Mantova N. S.", vol. 51, 1983, pp. 97-115.

C. Carducci, Ori e argenti dell'Italia antica, Roma 1962.

M. Dall'Acqua, Sculture mantovane d'età romanaBizzolano, Acquanegra, Casalmoro, in "Civiltà Mantovana”, vol. 23, 1969, pp. 329-354.

F. Ghedini, Nuovo ritratto di Livia al museo civico di Padova, in "Aquileia Nostra", vol. XLV- XLVI, 1975, pp. 361- 372.

G. A. Mansuelli, Le stele romae del territorio ravennate e del basso Po, Ravenna 1966.

F. Morandini - C. Stella, La città e le iscrizioni, in "Santa Giulia. Museo della città. L'età romana”, Brescia 1998, pp. 25-80.

F. Negretto, Monumenti funerari romani a edicola cuspidata del bolognese, in "Ocnus, Quaderni della Scuola di Specializzazione in Archeologia", $\mathrm{n}^{\circ} 12$, anno 2004, Bologna 2005, pp. 161- 198.

L. Pirzio Biroli Stefanelli, L'oro dei Romani. Gioielli di età imperiale, Roma 1992.

D. Scarpellini, Stele romane con imagines clipeatae in Italia, Roma 1987.

S. Stucchi, Il gruppo bronzeo tiberiano da Cartoceto, Roma 1988.

A. M. Tamassia, Cittadini mantovani d'età romana, in "Atti e memorie dell'Accademia Virgiliana di Mantova N. S., vol. 35, 1965, pp. 59-101.

A. M. Tamassia, Ritratto femminile dall'Antica Andes, in "Bollettino d'Arte", vol. 53, 1968, pp. 169-177.

A. M. Tamassia, Il commercio delle pietre nel mantovano in età romana, in "Annali Benacensi”, vol. 3, 1976, pp. 127-134.

A. M. Tamassia, Sculture romane dal Seminario

Diocesano di Mantova, in "Archeologia e Storia a Milano e nella Lombardia Orientale", Como 1980, pp. 137-155.

A. M. Tamassia, Riflessi della confisca dei terreni, in "Misurare la terra, il caso mantovano", Modena 1984, pp. 
TAMASSIA 1995

ZERBINATI 1982

ZEZZA 1982

ZILIANI 2001
89-94.

A. M. Tamassia, Una statua virile seduta, forse da Mantova, in "Splendida civitas nostra. Miscellanea di studi archeologici in onore di Antonio Frova", Roma 1995, pp. 281-283.

E. Zerbinati, Edizione archeologica della carta d'Italia al 100.000, foglio 64, Rovigo, Firenze 1982.

M. G. Zezza, I materiali lapidei locali impiegati in età romana nell'area compresa tra il Ticino e il Mincio, Milano 1982.

G. Ziliani, Il cippo di Septumia, una bambina romana, in "Quaderni di archeologia del mantovano", vol. 2, anno 2000, Mirandola 2001, pp. 91-99.

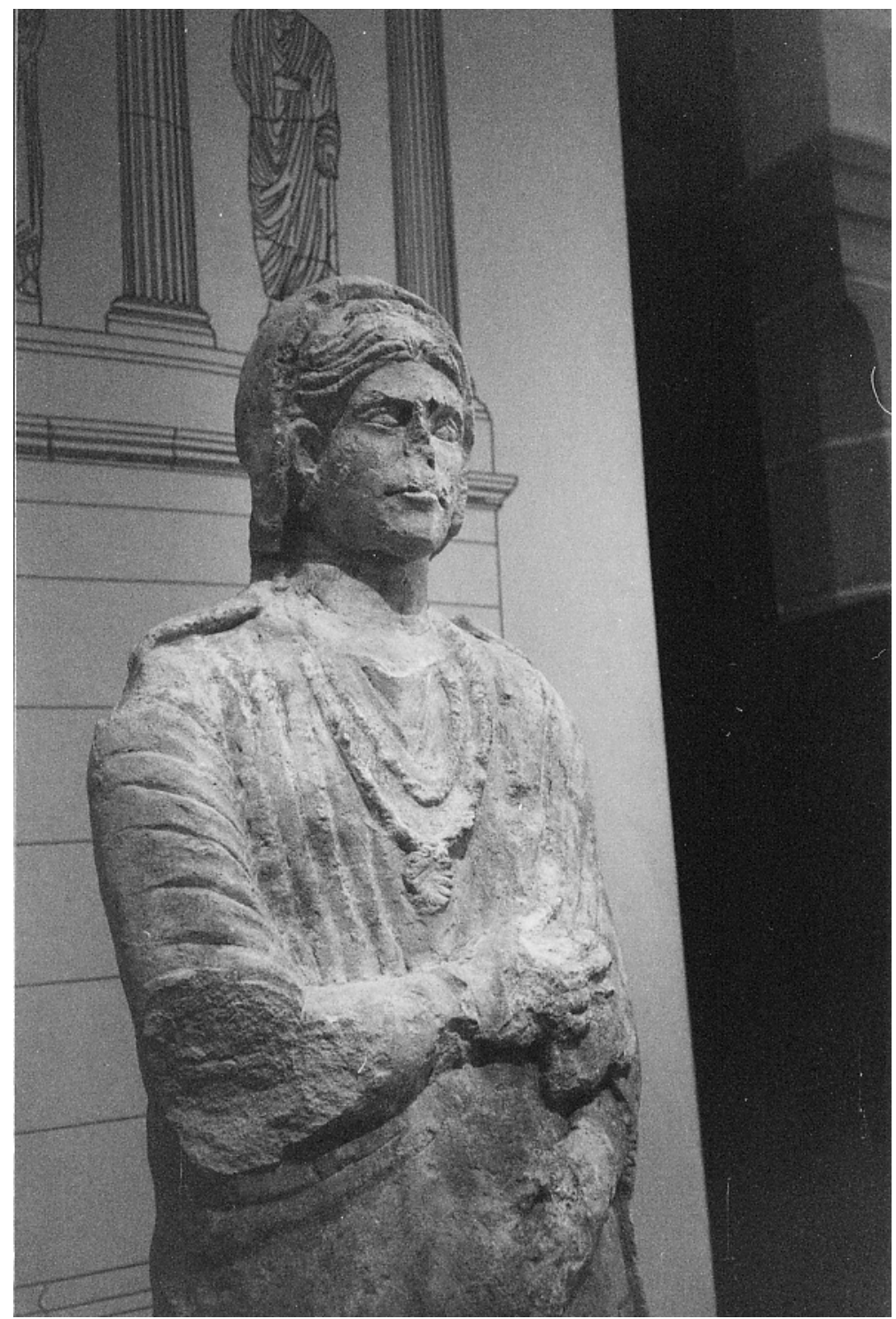

St. 11102 (su concessione del Ministero per i Beni e le Attività Culturali) 


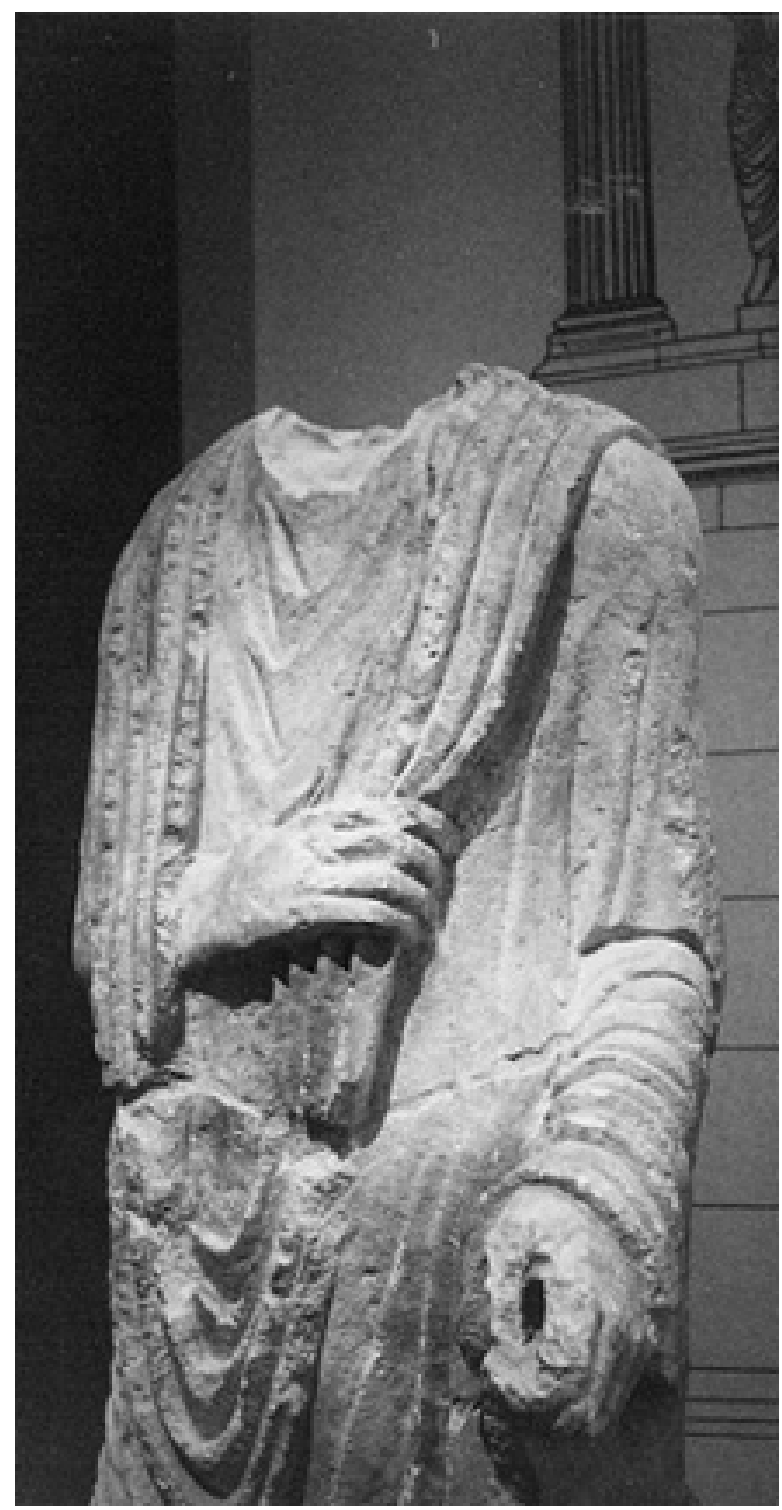

St. 11110 (su concessione del Ministero per i Beni e le Attività Culturali) 


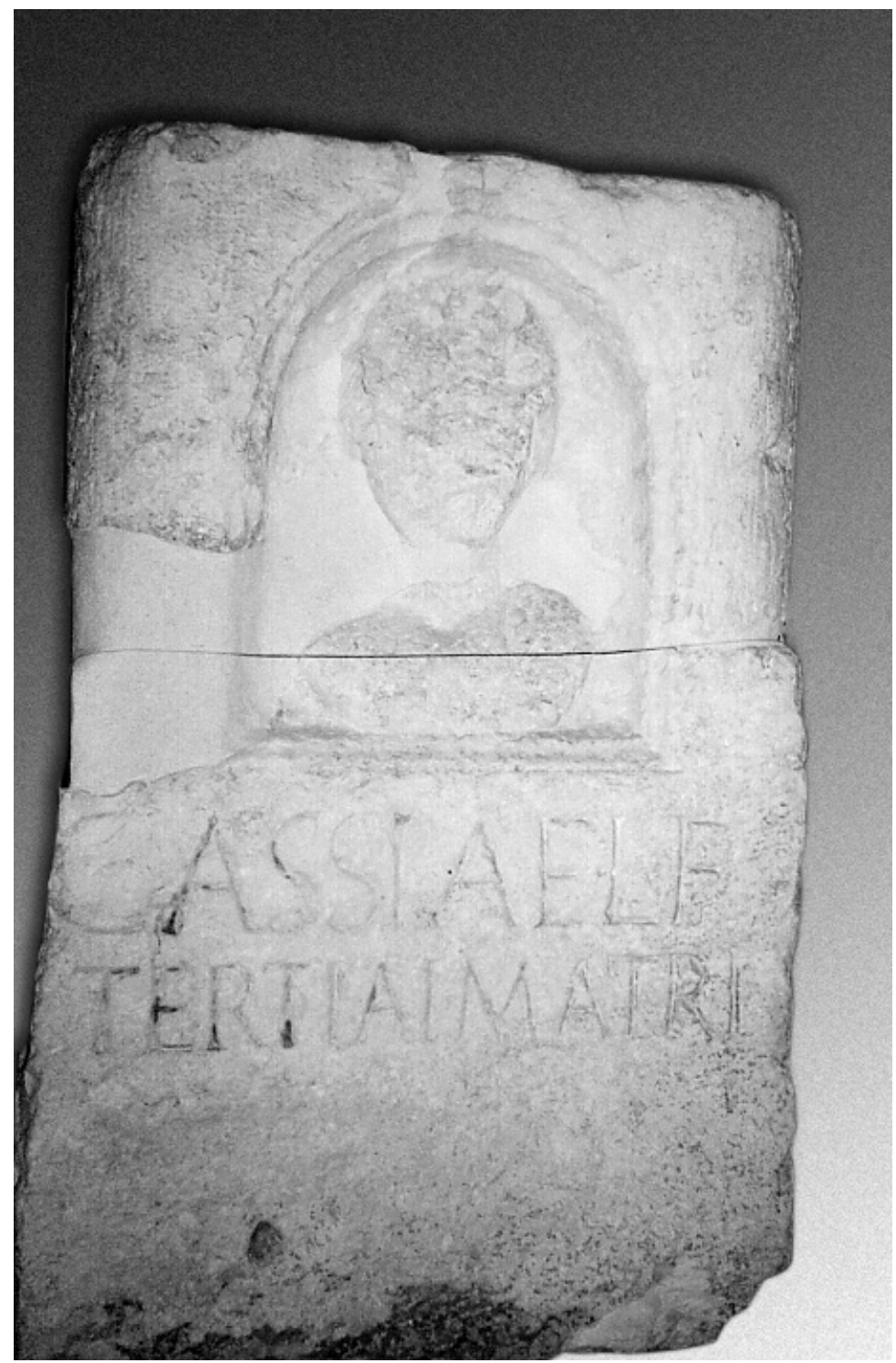

I.G. 12160 (su concessione del Ministero per i Beni e le Attività Culturali) 


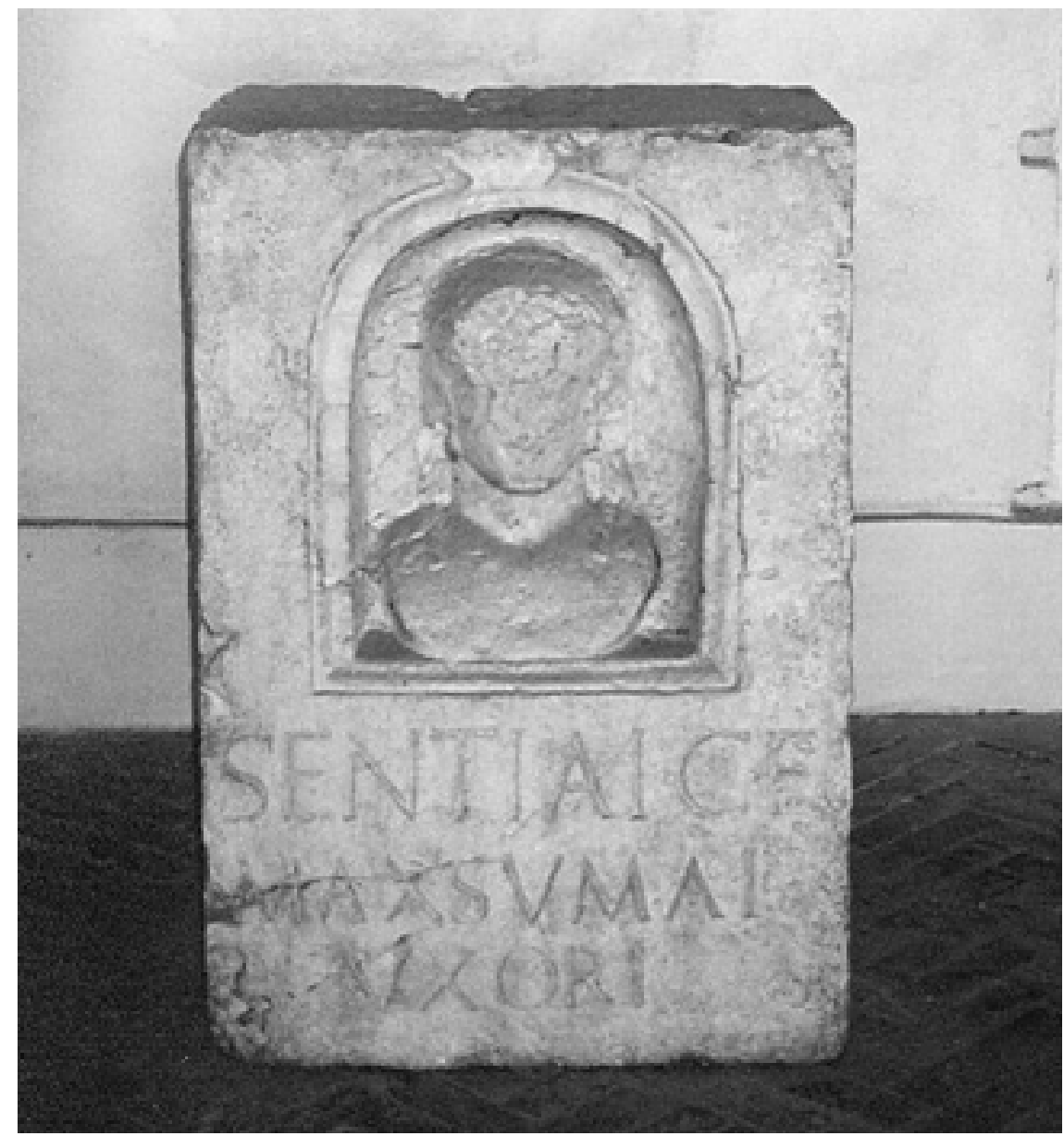

I.G. 12154 (su concessione del Ministero per i Beni e le Attività Culturali) 


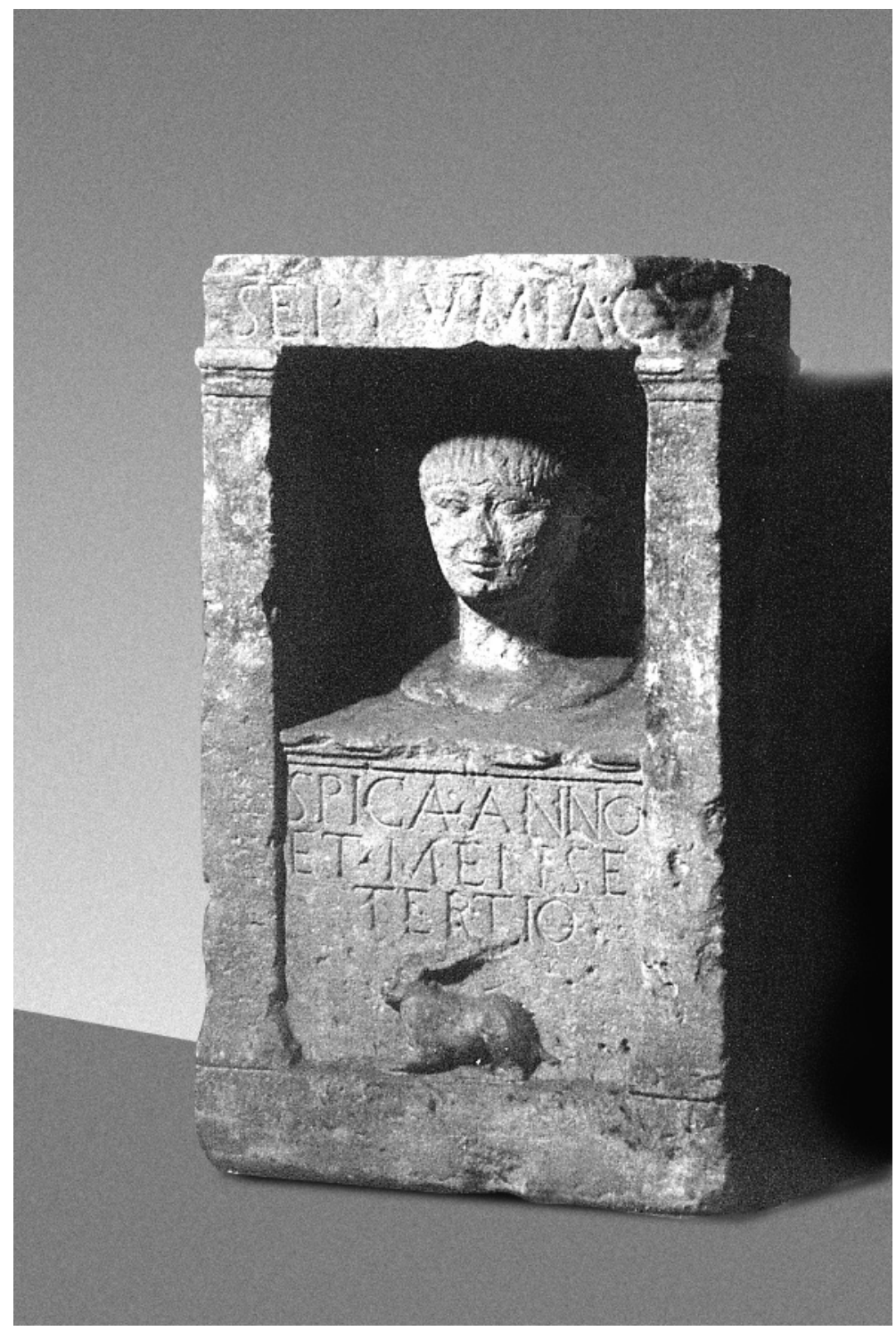

CIL V 2688 (su concessione del Ministero per i Beni e le Attività Culturali) 


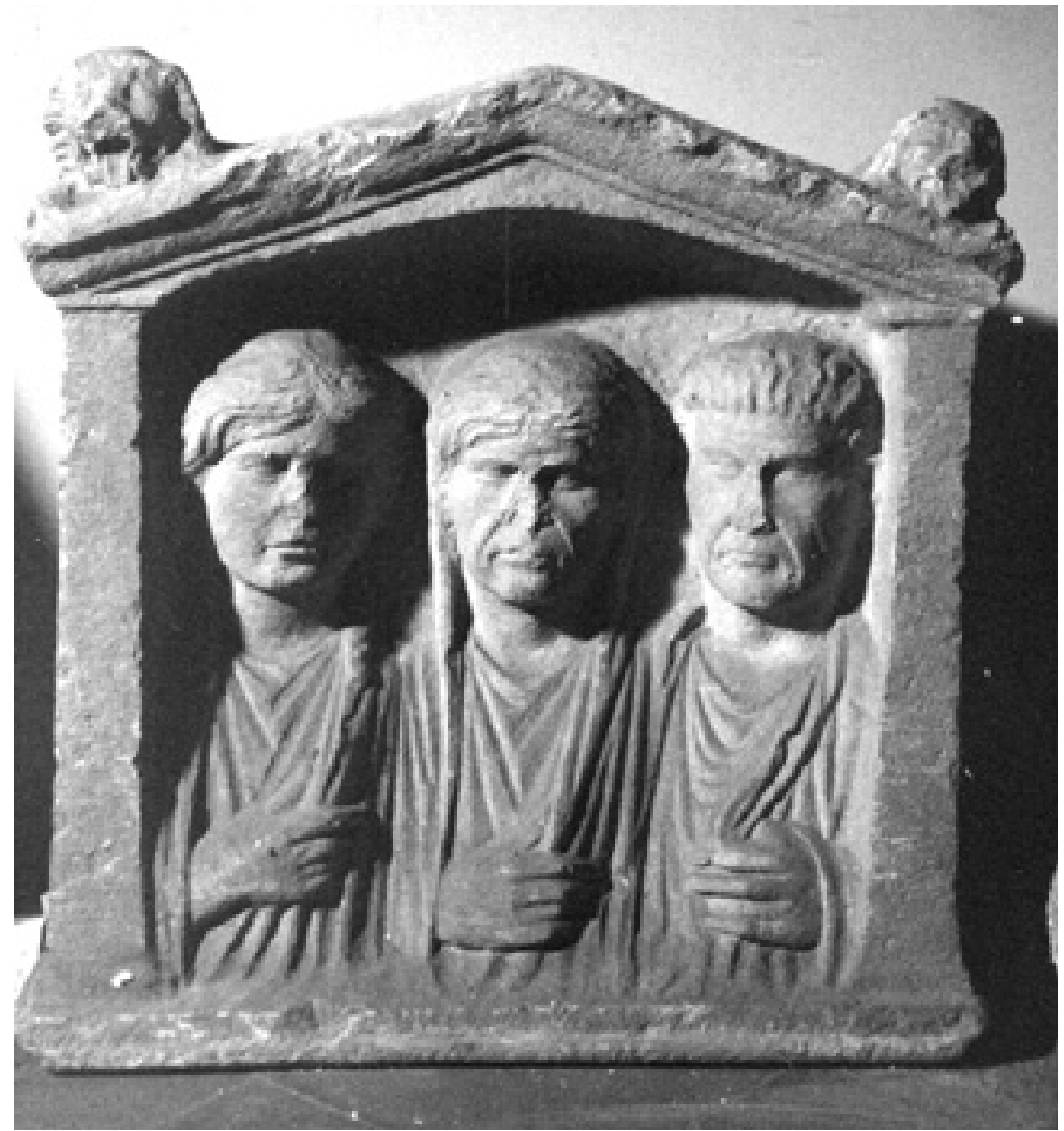

senza numero (su concessione del Ministero per i Beni e le Attività Culturali) 